\section{REPRESENTAÇÕES IDEATIVAS SOBRE EDENTULISMO E REABILITAÇÃO PROTÉTICA NA PERCEPÇÃO DE IDOSOS}

\author{
Ideational representations of edentulism and prosthetic \\ rehabilitation from the point of view of the elderly \\ Representaciones de ideas sobre el edentulismo y la \\ rehabilitación protética desde la perspectiva de mayores
}

Artigo Original

\section{RESUMO}

Objetivo: Identificar as representações ideativas de idosos edêntulos uni ou bimaxilares acerca das perdas dentárias e da reabilitação protética oral. Métodos: Estudo qualitativo, realizado entre janeiro e março de 2011 com sete idosos residentes em uma Instituição Pública de Longa Permanência do Recife-PE, com 14 idosos em atendimento na Clínica de Prótese Dentária da Universidade Federal de Pernambuco (UFPE). Coletaram-se os dados através de uma entrevista semiestruturada que passou por análise de conteúdo. Resultados: Os achados possibilitaram identificar que, para os idosos, os dentes contribuíam tanto para a saúde quanto como para facilitar interações sociais, enquanto o edentulismo foi associado a uma pluralidade de sentimentos negativos. Quanto à reabilitação protética, eles enfatizaram os prejuízos para a saúde devido a próteses mal adaptadas. Conclusão: Os idosos acreditam que o edentulismo e a reabilitação protética estão associados, principalmente, a um conceito mecanicista da profissão, amplamente difundido entre os profissionais que privilegiam mais a odontologia curativa em detrimento da prevenção. Nesse contexto, para que o envelhecimento possa ser considerado uma etapa da vida com as mesmas qualidades e dificuldades de qualquer outra, sugere-se aos gestores e aos próprios profissionais em saúde que se comprometam mais com uma prática odontológica humanizadora e preventiva, a fim de proverem os requisitos mínimos para um envelhecimento com dignidade.

Descritores: Idosos; Envelhecimento; Prótese Dentária; Reabilitação; Saúde Bucal; Qualidade de Vida.

\section{ABSTRACT}

Objective: To identify the ideational representations of upper and/or lower edentulous elders regarding tooth loss and oral prosthetic rehabilitation. Methods: Qualitative study conducted between January and March 2011 with seven elders living in a Public Longterm Care Institution in Recife, PE, and 14 elders attending the Dental Prosthesis Clinic of the Universidade Federal de Pernambuco - UFPE (Federal University of Pernambuco). Data were collected through semi-structured interview and underwent content analysis. Results: The results enabled to identify that, for the elders, the teeth contribute to health and facilitate the social interactions, while edentulism has been associated with a plurality of negative feelings. Regarding prosthetic rehabilitation, they emphasized the harms to health due to poorly fitted dental prosthesis. Conclusion: The elderly believe that edentulism and prosthetic rehabilitation are mainly associated with a mechanistic concept of the profession that is widely spread among the professionals who focus more on curative Dentistry at the expense of prevention. In this context, so that aging can be considered a stage of life with the same qualities and difficulties of any other stage, we suggest that managers and health professionals themselves should undertake a humanizing and preventive dental practice in order to provide the minimum requirements for an aging with dignity.

Descriptors: Elderly; Aging; Dental Prosthesis; Rehabilitation; Oral Health; Quality of Life.

\author{
Silvana Orestes-Cardoso ${ }^{(1)}$ \\ Kamilla Karla Maurício Passos ${ }^{(1)}$ \\ Saulo Lôbo Chateaubriand do \\ Nascimento(1) $^{(1)}$ \\ Milena Vieira da Silva Melo ${ }^{(1)}$ \\ Marilenede Oliveira Trindade ${ }^{(1)}$
}

1) Universidade Federal de Pernambuco UFPE - Recife (PE) - Brasil
Recebido em: 11/11/2014 Revisado em: 03/03/2015 Aceito em: 30/06/2015 


\section{RESUMEN}

Objetivo: Identificar las representaciones de ideas de mayores edentulos uni o bimaxilares sobre las pérdidas dentarias y de la rehabilitación protética oral. Métodos: Estudio cualitativo realizado entre enero y marzo de 2011 con siete mayores residentes de una Institución Pública de Larga Permanencia de Recife-PE con 14 mayores asistidos en la Clínica de Prótesis Dentaria de la Universidad Federal de Pernambuco (UFPE). Se recogieron los datos a través de una entrevista semi-estructurada que pasó por el análisis de contenido. Resultados: Los hallazgos posibilitaron identificar que para los mayores los dientes contribuyen para la salud y para facilitar las interacciones sociales mientras el edentulismo se asoció a una pluralidad de sentimientos negativos. Respecto la rehabilitación protética los mismos enfatizaron los perjuicios para la salud debido las prótesis mal adaptadas. Conclusión: Los mayores creen que el edentulismo y la rehabilitación protética se asocian principalmente al concepto mecanicista de la profesión muy difundido entre los profesionales que privilegian más a la odontología curativa que la prevención. En ese contexto, para que el envejecimiento pueda ser considerado una etapa de la vida con las mismas calidades y dificultades de cualquiera, sugiérase que los gestores y los propios profesionales de salud se involucren más con una práctica odontológica humana y preventiva para proveer los requisitos mínimos para el envejecimiento con dignidad.

Descriptores: Anciano; Envejecimiento; Prótesis Dental; Rehabilitación; Salud Bucal; Calidad de Vida.

\section{INTRODUÇÃO}

Estudos epidemiológicos realizados em diferentes países têm demonstrado que a população idosa, comparativamente a outras faixas etárias, representa o segmento populacional que obteve maior taxa de crescimento nos últimos $\operatorname{anos}^{(1,2)}$. Em parte, isso se deve, principalmente, a fatores como a diminuição da fecundidade e a redução da mortalidade, os quais têm assegurado o acesso de um maior número de indivíduos à terceira idade ${ }^{(2)}$. No Brasil, estimativas do Instituto Brasileiro de Geografia e Estatística já sinalizavam um aumento de $12,6 \%$ de idosos em 2013, para 25,1\% em 2025, o que elevará o país para a sexta posição mundial de indivíduos com idade igual ou superior a $60 \operatorname{anos}^{(3)}$.

A velhice é uma construção sociocultural, tendo a sua definição sofrido diversas transformações, de acordo com as diferentes culturas, ao longo do tempo ${ }^{(4)}$. Culturas como a chinesa, a árabe e a israelita dedicam respeito e veneração aos idosos, como meio de divulgação de sua história, sabedoria e experiência. No entanto, atualmente, observase na civilização ocidental a persistência de um enorme sentimento negativo em relação à velhice ${ }^{(5)}$.
Nesse contexto, em princípio, pode-se dizer que o conceito de idoso apresenta divergências entre os especialistas, porque, além de aspectos objetivos baseados em evidências mensuráveis, existem aspectos subjetivos que exercem bastante influência em relação à terceira idade. Existem autores que consideram idoso qualquer indivíduo com idade igual ou superior a $60 \operatorname{anos}^{(6)}$. No entanto, outros não acham apropriada a utilização apenas do critério cronológico para a identificação de pacientes geriátricos. Para eles, os idosos devem ser compreendidos em sua complexidade, uma vez que o conceito envolve aspectos biológicos, psicológicos, sociais, culturais e espirituais ${ }^{(7)}$.

Estudos realizados anteriormente evidenciaram que as representações sociais de velhice podem variar em diferentes grupos populacionais, de acordo com o sexo e a faixa etária ${ }^{(8)}$. No caso dos jovens, o idoso pode ser considerado uma pessoa impertinente, doente, cansada e solitária, enquanto para mulheres com mais de 50 anos, ele pode ser representado como uma pessoa tranquila e experiente, apesar das fragilidades orgânicas e psíquicas que possam apresentar.

A partir dos eixos temáticos investigados no presente estudo, é importante ressaltar que, no Brasil, para a população de idosos, os indicadores epidemiológicos em saúde bucal revelam indivíduos com lesões de mucosa, presença de grande quantidade de bolsas periodontais, edentulismo parcial ou total, assim como uso de próteses inadequadas, denunciadores de uma prática odontológica mutiladora $^{(1,9)}$. Os principais fatores etiológicos para as perdas dentárias são a cárie, seguida das doenças periodontais e dos traumatismos dentários ${ }^{(6,10,11)}$.

Dados do Ministério da Saúde acerca das condições de saúde bucal da população brasileira revelaram que, para a faixa etária de 65 a 74 anos, a porcentagem nacional de indivíduos usuários de prótese total no arco superior era de $63,7 \%$, e no inferior, de $37,5 \%$. Considerando-se a mesma faixa etária, o estudo também avaliou a necessidade de prótese dentária, sendo a prótese parcial em apenas um maxilar a de maior percentual nacional, $34,2 \%$. A prótese total bimaxilar se apresentou como necessária por $15,4 \%$ da população do país ${ }^{(9)}$,

Para que possa ser assegurado à população idosa um bem-estar biopsicossocial adequado, este deverá também contemplar a funcionalidade da mastigação, deglutição e fonação, além dos cuidados com a estética dentofacial, para a melhora tanto da autoestima como das interações sociais, minimizando, consequentemente, possíveis situações estigmatizadoras $^{(6,11,12)}$. É importante enfatizar que, tanto do ponto de vista estético quanto psicossocial, o significado da ausência dos dentes irá variar dependendo da relação que o idoso estabelece com seu corpo, e do grupo social ao qual pertence ${ }^{(6,13)}$. Nesse contexto, a saúde do sistema 
estomatognático contribui satisfatoriamente para que haja melhores integrações sociais e familiares, visto que a aparência, a fala, o sorriso e o beijo ficam comprometidos quando a condição bucal é inadequada ${ }^{(14)}$.

O equilíbrio biopsicossocial dos idosos deve integrar funções biológicas, emocionais e espirituais. Dependendo do modo como essas funções estão interrelacionadas, qualquer mudança em uma delas pode causar repercussões nas outras ${ }^{(15)}$

O fato de se considerar a dinâmica psíquica do idoso rica e complexa, principalmente devido às suas dificuldades adaptativas tanto emocionais quanto fisiológicas ${ }^{(16)}$, não significa que o processo de envelhecimento seja sinônimo de doença, uma vez que muitas desordens orgânicas e emocionais podem ser prevenidas nessa faixa etária ${ }^{(17)}$.

Dessa maneira, envelhecer de forma saudável significa que, além de um bom estado de saúde física, os idosos necessitam de respeito, segurança e oportunidades para expressar livremente seus sentimentos, emoções, interesses, opiniões e experiências ${ }^{(8)}$.

Uma das alterações emocionais frequentes no processo de envelhecimento consiste na labilidade afetiva, caracterizada por mudanças rápidas nas emoções, variando clinicamente da hiapomania a explosões de humor ou manifestações de cólera diante de estímulos insignificantes $^{(7,18,19)}$.

As emoções positivas vivenciadas ao longo da vida protegem o sistema imunológico, ajudando o corpo a combater as infecções ${ }^{(8)}$. Portanto, é oportuno enfatizar os efeitos do bom humor e dos bons sentimentos para a qualidade de vida e na saúde global das pessoas ${ }^{(13)}$.

Tendo em vista as características psicossociais e o perfil epidemiológico de saúde bucal dos brasileiros acima de 60 anos, partimos da premissa de que, para essa população, o edentulismo e a utilização de próteses são simbolizados como consequências naturais do processo de envelhecimento.

As representações que os idosos fazem de seus sentimentos em relação às categorias investigadas ainda não foram suficientemente exploradas pela odontogeriatria. Estudos dessa natureza são importantes porque possibilitam uma compreensão qualitativa e ampliada do conceito de saúde ao longo do processo de envelhecimento.

Adotando-se essa linha de reflexão, o presente estudo teve por objetivo identificar as representações ideativas de idosos edêntulos uni ou bimaxilares acerca das perdas dentárias e da reabilitação protética oral.

\section{MÉTODOS}

Trata-se de uma pesquisa qualitativa, realizada com sete idosas do Abrigo Espírita Lar de Jesus - Associação
Filantrópica e 14 idosos (sete homens e sete mulheres) em atendimento na Clínica de Prótese Dentária da Universidade Federal de Pernambuco (UFPE). Convém salientar que na cidade do Recife-PE não existem Instituições de Longa Permanência para o sexo masculino, tanto na rede pública quanto naqueles subvencionados por associações filantrópicas. Excluíram-se da pesquisa os idosos que apresentavam dificuldades de expressão e comunicação. A definição do número de indivíduos não se fez com base em amostragem probabilística, sendo o número de sujeitos delimitado pela saturação das falas ${ }^{(20)}$

Pesquisarealizadaentrejaneiroemarçode2011.Utilizouse para coleta de dados uma entrevista semiestruturada, para obtenção de variáveis socioeconômico-demográficas (sexo, idade, cor, estado civil, procedência, renda individual e escolaridade) e clínicas (tempo de perda do último dente, de utilização e de confecção da prótese total) dos pacientes. As questões abertas foram: Por que motivo o(a) $\operatorname{Sr}(a)$. perdeu todos os dentes? Qual a importância deles para o(a) Sr(a).? Como se sentiu ao perdê-los? Quais os sofrimentos de não se ter dentes? Que mudanças ocorreram em sua vida com a perda? Adquiriu algum problema de saúde por utilizar prótese? Quais os benefícios e as dificuldades de utilizá-la? Está satisfeito(a) com ela?

Para a organização dos dados, foi utilizado o método de análise de conteúdo ${ }^{(21)}$, o qual pode ser dividido em três fases: pré-análise, exploração dos resultados e interpretação das informações. A pré-análise é a própria organização do trabalho. É nessa fase que se faz a escolha do objeto de estudo, bem como a formulação dos objetivos do trabalho. Estando decidido o que estudar, é necessário proceder à constituição do corpus. Corpus nada mais é que o conjunto do material que será submetido a uma análise. A exploração do material consiste em uma fase longa, com procedimentos de codificação ou enumeração em função de regras previamente formuladas. A última fase do processo de análise de conteúdo é a interpretação dos resultados, que se baseia literalmente em interpretar os resultados encontrados $^{(21)}$.

Após a transcrição das entrevistas gravadas, as informações obtidas foram exaustivamente lidas, sintetizadas e categorizadas em quatro categorias temáticas: Motivo da perda de dentes; Importância da perda dos dentes; Sentimentos e mudanças na vida após a perda dos dentes; Benefícios e dificuldades de utilizar a prótese dentária, as quais foram confrontadas com os referenciais teóricos previamente selecionados.

A pesquisa teve aprovação pelo Comitê de Ética e Pesquisa em Seres Humanos do Centro de Ciências da Saúde da Universidade Federal de Pernambuco (CCS/ UFPE), CAAE - 0096.0.172.000-09. Todos os participantes concordaram em assinar o Termo de Consentimento Livre 
e Esclarecido e, para que o anonimato fosse preservado, utilizaram-se codinomes de filósofos para os homens, e de deusas da mitologia grega para as mulheres.

\section{RESULTADOS E DISCUSSÃO}

Inicialmente, apresentaram-se os dados sociodemográficos, econômicos e clínicos dos entrevistados, para em seguida serem apresentadas as categorias temáticas emergidas do estudo.

Através de um formulário estruturado, constatouse que os 21 idosos que participaram da pesquisa eram predominantemente do sexo feminino, encontravam-se na faixa etária entre 60 e 69 anos, autodenominavamse leucodermas, eram solteiros, procedentes da Região Metropolitana do Recife, aposentados, com renda individual de um salário mínimo e não tinham completado o ensino fundamental.

Considerando-se a amostra total, a maioria dos entrevistados tinha perdido o último dente entre 30 e 59 anos, eram usuários de prótese total há mais de 20 anos e estavam utilizando a prótese pela primeira vez, tendo sido confeccionada no período compreendido entre 1 e 5 anos.

A história da odontologia no Brasil remete para um quadro epidemiológico de alta prevalência de edentulismo ${ }^{(1,9,16)}$, principalmente entre os idosos, refletindo, a princípio, um modelo de atenção odontológica cirúrgicorestaurador responsável por inúmeras exodontias e posterior utilização de próteses totais ${ }^{(6)}$.

\section{Motivo da perda de dentes}

Ao se investigar os motivos pelos quais os entrevistados tinham se tornado edêntulos totais, constatou-se que as perdas dentárias foram em decorrência, principalmente, da evolução do processo carioso, da doença periodontal e de traumatismo dentário.

A seguir, estão transcritas algumas justificativas apresentadas para as perdas dentárias:

"Os dentes ficaram estragados, furados, fracos $e$ doendo." (Afrodite)

“As obturações caíam.” (Platão)

"No passado, os dentistas não se preocupavam com essa história de prevenção.” (Aristóteles)

"Essa história de perder dente é uma coisa de pai para filho." (Hegel)

No contexto do presente estudo, a maioria das elaborações apresentadas pelos entrevistados para o edentulismo pôde ser categorizada em dois grandes grupos. O primeiro, constituído de justificativas mais realistas, pois conseguiam identificar que, se os dentes ficaram cariados, isso ocorreu, principalmente, por negligência deles próprios, por falta de uma odontologia centrada na prevenção e/ou por dificuldade de acesso aos serviços odontológicos, entre outros motivos. Nesse sentido, as percepções que tinham para as perdas dentárias reforçam o consenso existente na literatura de que as ações de cuidado à saúde bucal, historicamente, têm sido estruturadas como uma prática caracterizada pelo curativismo ${ }^{(6,11)}$.

No segundo grupo, foram inseridas as justificativas menos investigadas pelos autores consultados, visto serem mais emocionais e forjadas pelo imaginário popular, através de crenças e mitos introjetados, a exemplo de ser natural que os dentes fiquem cariados ou de existir uma predisposição hereditária para se ter dentes fracos. Esse conjunto de crenças pode ser avaliado, ainda, do ponto de vista da identidade cultural e racial do indivíduo, cabendo a alguns grupos étnicos a ideação de maior resistência e a outros, uma fraqueza resignada.

\section{Importância da perda dos dentes}

Quando perguntados sobre a importância dos dentes, responderam:

"Eles servem para a pessoa ter uma boa digestão." (Spinoza)

"Sentir o gosto dos alimentos." (Nietzsche)

“Não ficar com mau hálito.” (Atena)

A mesma dissimetria observada para as justificativas apresentadas por eles acerca do edentulismo também foi observada em relação à importância dos dentes para o bemestar biopsicossocial. Se por um lado, predominantemente, eles associavam a dentição natural à preservação da mastigação, digestão, estética, fonação e interação social satisfatórias, funções estas amplamente discutidas na literatura em decorrência da perda dos dentes e da utilização de próteses dentárias parciais e/ou totais ${ }^{(5,11,17)}$, incluindose também a alusão que faziam da importância dos dentes para morder, triturar e roer objetos, funções estas bastante primárias e que remontam aos primeiros anos de vida do sujeito; por outro lado, persistiam algumas crenças de que os dentes eram responsáveis pelo paladar, e que a ausência deles provocava halitose.

Quanto a essas crenças, merece destaque que, para eles, o fato de exercerem com dificuldade o ato fisiológico de triturar e roer os alimentos para poder saboreá-los interferia negativamente no prazer que sentiam de se alimentarem. Isso se justifica, clinicamente, pela necessidade que os edêntulos ou usuários de próteses totais têm de selecionar alimentos ou utilizar outras estratégias que facilitem a ingestão, como a trituração prévia. Outro agravo associado à perda dos 
dentes consiste na redução fisiológica do fluxo salivar, que diminui a capacidade de deglutição dos alimentos ${ }^{(16)}$.

A mudança de uma dieta saudável para uma com predominância de carboidratos e alimentos menos consistentes pode não conter os nutrientes adequados às necessidades biológicas, causando estados anêmicos e apáticos em pessoas mais suscetíveis. Além disso, esse tipo de alimentação pode causar atrofia na musculatura mastigatória, com repercussão na estética facial e na autoestima do idoso ${ }^{(16)}$.

\section{Sentimentos e mudanças na vida após a perda dos dentes}

Quando questionados acerca dos sentimentos que emergiram neles após a perda de todos os dentes, as respostas mais frequentemente relatadas foram:

"Fiquei triste quando perdi os dentes. Eu vejo as pessoas por aí com os dentes, sorrindo, é bonito, não é? A pessoa sem poder rir para os outros e sem comer direito, a vida fica sem graça.” (Sartre)

"Eu me senti arrasada, porque a prótese não é nunca como a gente ter os dentes da gente. A pessoa perde até o gosto da comida. Tudo é ruim." (Dione)

Tendo em vista a pluralidade de sentimentos de valência negativa identificados nas falas dos entrevistados, a exemplo de tristeza, culpa, vergonha, humilhação, baixa autoestima, dentre outros, merece destaque o fato de que o processo de envelhecimento envolve mudanças fisiológicas (perdas das acuidades visual e auditiva, do tônus muscular, alterações metabólicas, entre outras) e psicossociais (perda de status, isolamento social, sentimento de inutilidade etc.), as quais interferem na qualidade de vida do sujeito. A condição de edentulismo em pessoas idosas pode evoluir para quadros depressivos que variam de $10 \%$ a $24 \%$ para as não institucionalizadas e de $25 \%$ a $80 \%$ para as institucionalizadas ${ }^{(17,22-24)}$.

É importante enfatizar que todas essas características, frequentemente encontradas nos idosos, agravam-se quando os pacientes são edêntulos, pois a redução na qualidade de vida é ainda mais acentuada. Por outro lado, é pertinente destacar que, sendo a cavidade oral fonte de nutrição e zona erógena por excelência ${ }^{(25)}$, neste estudo, é muito significativa a dicotomia que existe entre os sexos para justificarem as perdas dentárias. No grupo das mulheres, institucionalizadas ou não, dentes que iam "se quebrando, doíam, ficavam moles, furados, fracos, doentes, estragados, caíam e se rachavam" não prestavam para a mastigação, e desse modo, "a boca ficava murcha". No grupo dos homens, os dentes estiveram mais associados aos conceitos de virilidade, remetendo a uma visão pragmática da situação, uma vez que dentes com "buracos, moles, velhos e cansados" precisavam ser "arrancados".
Os entrevistados relataram como mudanças mais frequentes:

"Tenho dificuldade de me alimentar, porque para comer preciso passar a comida no liquidificador. Se eu não mastigo direito, meu estômago e meu esôfago trabalham mais. Isso prejudica minha saúde." (Aristóteles)

"Sem os dentes, fica dificil sorrir. E é ruim até para beijar." (Nietzsche)

Com relação aos problemas relatados associados ao edentulismo, a exemplo de saúde, estética e fala prejudicadas, merece destaque, pela frequência dos relatos, a dificuldade para a mastigação, uma vez que, além de prejudicar a saúde, gerava ansiedade, impaciência e incômodo, porque eram necessárias mudanças nos hábitos alimentares para se adequarem à condição de edêntulos. Desse modo, o ato de se alimentar deixava de ser prazeroso

Há consenso entre os autores consultados, ao abordarem a importância estético-funcional dos dentes, que o edentulismo, além de interferir em funções vitais para o indivíduo e acarretar prejuízos para a saúde, diminui a sensação de bem-estar ${ }^{(23,26-28)}$.

Quando interrogados sobre as mudanças que ocorreram em suas vidas devido às perdas dentárias, as respostas puderam ser agrupadas da seguinte maneira: alterações nos hábitos alimentares - por dificuldades em cortar, morder e triturar os alimentos, geradores de "impaciência, nervosismo, ansiedade, irritabilidade e agonia na boca" e sociais (evitar interagir com os outros); e transformação na imagem corporal, com consequente sentimentos de vergonha, constrangimento, inferioridade e isolamento social, devido à perda das estéticas facial e do sorriso, pois:

"Sem os dentes, a gente se sente um lixo, um bagulho." (Afrodite)

Especificamente no grupo dos homens, alguns responderam que as mudanças foram poucas, pois não dependiam da aparência para sobreviver; além do mais, extrair dentes estragados e colocar uma prótese era um benefício para a saúde:

"Porque eu vivia sentindo dor de ouvido, dor de cabeça, dor na vista. Agora, graças a Deus, consegui a prótese!" (Spinoza)

Enfatizamos que o ato de mastigar é uma das mais importantes funções orais, e a capacidade mastigatória dos usuários de prótese total pode ficar reduzida em até $25 \%{ }^{(29)}$. Por isso, os idosos tendem a buscar alimentos mais maleáveis, pois percebem que a mastigação não apresenta mais a mesma eficácia ${ }^{(1)}$. A perda de dentes, aliada à diminuição do fluxo salivar, no idoso, favorece a diminuição 
da capacidade mastigatória, causando comprometimentos para a saúde geral e o bem-estar psicossocial ${ }^{(6)}$.

\section{Satisfação, benefícios e dificuldades acarretadas ao utilizar a prótese dentária}

Nesta categoria, os relatos dos entrevistados sobre o nível de satisfação com o aparelho protético que estavam utilizando foram predominantemente positivos, porque a prótese estava bem adaptada, não machucava, eles podiam mastigar, sorrir e interagir com os outros, sem medo. No entanto, alguns afirmaram estar insatisfeitos devido à falta de retenção e de estabilidade.

Constatou-se, portanto, que o nível de satisfação esteve associado a aspectos funcionais (possibilidade de mastigar melhor e conforto no uso da prótese) e sociais (melhor interação social). Nesse contexto, desde que os dentes artificiais atendam às expectativas dos sujeitos, seu uso é capaz de melhorar a autoestima e as relações interpessoais ${ }^{(6)}$. Tendo em vista que o ato da alimentação pode ser associado à obtenção de prazer, os indivíduos edêntulos encontrar-seiam, portanto, privados desse tipo de satisfação, e as próteses podem se tornar um meio de amenizar este déficit ${ }^{(1)}$.

Quanto aos benefícios de utilizarem uma prótese, verificamos que estes se relacionavam a melhoras relativas à mastigação, estética, autoestima, fala, nos relacionamentos interpessoais e na possibilidade de recuperação, através de próteses, da imagem corporal perdida devido ao edentulismo.

No que diz respeito a esses benefícios, confrontando nossos resultados com a literatura, foi possível inferir que o edentulismo pode causar transtornos biopsicossociais significativos $^{(13,26,27)}$; o que torna evidente a complexidade da reabilitação protética, tanto para o restabelecimento de funções vitais (como a fala e a mastigação) quanto para a preservação da qualidade de vida nos idosos.

No que diz respeito às dificuldades relativas ao uso de prótese total, dentre aqueles que relataram prejuízo para a saúde, constatou-se que:

"Uma prótese mal adaptada provoca ulcerações na gengiva e estomatite." (Íris)

"Esta prótese está me deixando com dor de ouvido." (Hegel)

"Como eu não mastigo direito, fico com gastrite." (Sócrates)

Com relação a esse aspecto, a literatura consultada reforça nossos resultados, uma vez que o cirurgiãodentista deve se preocupar com vários aspectos ao planejar uma prótese dentária, dentre eles, com a função da articulação temporomandibular (ATM), saúde da mucosa oral, tonicidade da musculatura, higiene oral e da prótese, condições oclusais, forma e tamanho do rebordo, distribuição das forças mastigatórias e condição sistêmica do paciente ${ }^{(30)}$. Merece destaque o fato de que grande parte dos cirurgiões-dentistas perde o interesse pelo paciente depois de instalada a prótese, o que compromete o prognóstico do tratamento, visto que essa atitude influencia negativamente na manutenção da saúde oral( ${ }^{(31)}$.

Diferentes estudos têm demonstrado a grande prevalência de transtornos causados por problemas técnicos na confecção da prótese, assim como pela falta de manutenção e acompanhamento da condição oral durante o uso desta ${ }^{(32-34)}$.

As dificuldades mais frequentes relatadas quanto ao uso de próteses foram relacionadas com a mastigação, a comunicação e a adaptação à prótese, principalmente quando esta não havia sido confeccionada com todo o rigor técnico e científico. Alguns autores ${ }^{(6,35)}$ ressaltam como principal dificuldade a estética deficiente, enquanto para outros $^{(1,13)}$, é confirmada a importância do ato mastigatório para o bem-estar físico e psicológico.

\section{CONSIDERAÇÕES FINAIS}

As concepções ideativas identificadas pelos pacientes entrevistados, tanto em relação à sua condição de edêntulos quanto a de usuários de próteses totais, estavam amplamente permeadas por uma odontologia de caráter eminentemente mecanicista e muito pouco humanista, consequentemente, mais centrada no tratamento de processos patológicos do que na prevenção e educação, desconsiderando aspectos como transdisciplinaridade e condições psicossociais específicas de sujeitos idosos. Os resultados obtidos, infelizmente, confirmam o pressuposto inicial de que a perda dos dentes foi considerada um processo natural do envelhecimento.

Apesar da dificuldade de se conseguir que os entrevistados expressassem livremente seus sentimentos, emoções, percepções, interesses, opiniões e experiências em relação às categorias investigadas, consideramos relevante destacar o quanto é promissor, para que possam assumir o compromisso de cuidarem da própria saúde e terem uma melhor qualidade de vida, que indivíduos nessa faixa etária considerem as condições relativas ao edentulismo e à má adaptação de próteses como fatores que interferem nos prazeres do dia a dia, como saborear, sorrir e beijar.

Diante do exposto, e para que o envelhecimento possa ser considerado uma etapa da vida com as mesmas qualidades e dificuldades de qualquer outra, sugerimos aos gestores e aos próprios profissionais em saúde que se comprometam mais com uma prática odontológica humanizadora, a fim de proverem os requisitos mínimos para um envelhecimento com dignidade. 


\section{REFERÊNCIAS}

1. Piuvezam G, Ferreira AAA, Soares SCM, Souza DLB, Alves MSCL. As perdas dentárias sob a ótica do idoso. Odontol Clín-Cient. 2006;5(4):299-306.

2. Veras R. Envelhecimento populacional contemporâneo: demandas, desafios e inovações. Rev Saúde Públ. 2009;43(3):548-54.

3. Instituto Brasileiro de Geografia e Estatística IBGE. Censo Demográfico 2010 [acesso em 2014 Set 20] Disponível em: http://www.ibge.gov. br/home/estatistica/populacao/condicaodevida/ indicadoresminimos/sinteseindicsociais2013/default tab_xls.shtm.

4. Beauvoir S. A velhice. Rio de Janeiro: Nova Fronteira; 1990.

5. Freitas AS, Costa MJ. A identidade social do idoso: memória e cultura popular. Rev Conexão UEPG. 2011;7(2):202-11.

6. Unfer B, Braun K, Silva CP, Pereira Filho LD, Autopercepção da perda de dentes em idosos. Interface Comun Saúde Educ. 2006;10(19):217-26.

7. Santos SSC. Concepções teórico-filosóficas sobre envelhecimento, velhice, idoso e enfermagem gerontogeriátrica. Rev Bras Enferm. 2010;63(6):103539.

8. Silva DD, Sousa MLR, Wada RS. Autopercepção e condições de saúde bucal em uma população de idosos. Cad Saúde Pública. 2005;21(4):1251-9.

9. Ministério da Saúde (BR). Projeto SB Brasil 2010. Pesquisa nacional de saúde bucal. Resultados Principais. Brasília: Ministério da Saúde; 2012.

10. Moreira RS, Nico LS, Tomita NE. O risco espacial e fatores associados ao edentulismo em idosos em município do sudeste do Brasil. Cad Saúde Pública. 2011;27(10):2041-53.

11. Narvai PC, Antunes JLF. Saúde bucal: a autopercepção da mutilação e das incapacidades. In: Lebrão ML, Duarte TAO. SABE no município de São Paulo: uma abordagem inicial. Brasília: Editora da OPAS; 2003. p. 121-40.

12. Kimura C. Por que idosos desdentados precisam do dentista? Rev Portal Divulg. 2011;13: 7-11.

13. Pesquero ACB. Uso de prótese dentária total por idosos: aspectos psicológicos [tese]. Goiânia: Universidade Católica de Goiás; 2005.
14. Musacchio EM, Perissinotto E, Binotto P, Sartori L, Silva-Neto F, Zambon S, et al. Tooth loss in the elderly and its association with nutritional status, socioeconomic and lifestyle factors. Acta Odontol Scand. 2007;65(2):78-86.

15. Maciel ACM, Guerra RO. Influência dos fatores biopsicossociais sobre a capacidade funcional de idosos residentes no nordeste do Brasil. Rev Bras Epidemio. 2007;10(2):178-89.

16. Montenegro FLB, Brunetti RF, Manetta CE. Interações entre a Medicina e a Odontologia. In: Brunetti RF, Montenegro FLB. Odontogeriatria: noções de interesse clínico. São Paulo: Artes Médicas; 2002. p. 99-114.

17. Silva EMM, Silva Filho CE, Fajardo RS, Fernandes AUR, Marchiori AV. Mudanças fisiológicas e psicológicas na velhice relevantes no tratamento odontológico. Rev Ciênc Ext. 2005;2(1):63.

18. Aas M, Aminoff SR, Lagerberg TV, Etain B, Agartz I, Andreassen AO, et al. Affective lability in patients with bipolar disorders is associated with high levels of childhood trauma. Psychiatry Res. 2014;218(1-2): 252-5.

19. Aminoff SR, Jensen J, Lagerberg TV, Hellvin T, Sundet $\mathrm{K}$, Andreassen $\mathrm{OA}$, et al. An association between affective lability and executive functioning in bipolar disorder. Psychiatry Res. 2012;198(1):58-61.

20. Bauer MW, Aarts B. A construção de corpos: um princípio para coleta de dados qualitativos. In Bauer MW, Gaskell G, editores. Pesquisa qualitativa com texto, imagem e som. 11 ${ }^{\mathrm{a}}$ ed. Petrópolis: Vozes; 2013. p. 39-63.

21. Bardin L. Análise de conteúdo. São Paulo: Edições 70; 2011.

22. Smith JM, Sheiham A. How dental conditions handicap the elderly. Community Dent Oral Epidemiol. 1979;7(6):305-10.

23. Fiske J, Davis DM, Frances C, Gelbier S. The emotional effects of tooth loss on edentulous people. Br Dent J. 1998;184(2):90-3.

24. Pena FB, Santo FHE. O movimento das emoções na vida dos idosos: Um estudo com um grupo da terceira idade. Rev Eletrônica Enferm. 2006;8(1):17-24.

25. Freud S. Três ensaios sobre a teoria da sexualidade. In: Edição Standard Brasileira das Obras Psicológicas Completas de Sigmund Freud. Rio de Janeiro: Imago; 2009. p. 119-229. 
26. Strauss RP, Hunt RJ. Understanding the value of teeth to older adults: influences of quality of life. JADA. 1993;124(1):105-10.

27. Nakata M. Mastigatory function and its effects on general health. Int Dent J. 1998;48(6):540-8.

28. Scott BJJ, Leung KCM, Mcmillan AS, David DM, Fiske J. A transcultural perspective on the emotional effect of tooth loss in complete denture wearers. Int $\mathrm{J}$ Prosthodont. 2001;14(5):461-5.

29. Moriguchi Y. Aspectos geriátricos no atendimento odontológico. Odontol Mod. 1992;19(4):11-3.

30. Turano JC, Turano LM, Turano MV. Fundamentos de prótese total. $9^{\mathrm{a}}$ ed. São Paulo: Santos; 2012.

31. Wendt D. How to promote and maintain good oral health in spite of wearing dentures. J Prosthet Dent. 1985;53(6):805-7.

32. Medeiros JJ, Rodrigues LV, Azevedo AC, Lima Neto EA, Machado LS, Valença AMG. Edentulismo, uso e necessidade de prótese e fatores associados em município do Nordeste brasileiro. Pesq Bras Odontoped Clin Integr. 2012;12(4):573-8.
33. De Carli JP, Giaretta BM, Vieira RR, Linden MSS, Ghizoni JS, Pereira JR. Lesões bucais relacionadas ao uso de próteses dentárias removíveis. Salusvita. 2013;32(1):103-15.

34. Al-Imam H, Özhayat EB, Benetti AR, Pedersen AML, Gotfredsen K. Oral health-related quality of life and complications after treatment with partial removable dental prosthesis. J Oral Rehabil. 2015;42(8):1-8.

35. Roumanas ED. The solution-denture esthetics, phonetics and function. J Prosthodont. 2009;18(2): 112-5.

\section{Endereço para correspondência:}

Silvana Orestes Cardoso

Rua Djalma Farias, 29

Bairro: Torreão

CEP 52030-190 - Recife - PE - Brasil

E-mail: silvanaorestes@hotmail.com 